\title{
Carotid artery patch plasty as a last resort repair for long-segment congenital tracheal stenosis
}

Ali Dodge-Khatami, MD, ${ }^{a}$ Nic C. Nijdam, MD, PhD, ${ }^{b}$ Eli Broekhuis, MD, ${ }^{a}$ Ines A. von Rosenstiel, MD, ${ }^{c}$ Peter G. Dahlem, MD, ${ }^{c}$ and Mark G. Hazekamp, MD, PhD, ${ }^{a}$ Amsterdam, The Netherlands

From the Divisions of Cardiothoracic Surgery, ${ }^{\mathrm{a}}$ Otorhinolaryngology, ${ }^{\mathrm{b}}$ and Pediatric Intensive Care, ${ }^{\mathrm{C}}$ Academic Medical Center, University of Amsterdam, Amsterdam, The Netherlands.

Received for publication Oct 18, 2001; accepted for publication Nov 5, 2001.

Address for reprints: A. Dodge-Khatami, MD, Afdeling Cardiothoracale Chirurgie, Academic Medical Center-Postbus 22660, 1100 DD Amsterdam, The Netherlands (E-mail: a.dodgekhatami@amc.uva.nl).

J Thorac Cardiovasc Surg 2002;123:826-8

Copyright $(02002$ by The American Association for Thoracic Surgery

$0022-5223 / 2002 \$ 35.00+0 \quad \mathbf{1 2 / 5 4 / 1 2 2 0 6 5}$

doi: $10.1067 / \mathrm{mtc} .2002 .122065$
A previously healthy 4-month-old girl had acute respiratory distress and exhaustion, mandating intubation and mechanical ventilation. The chest $\mathrm{x}$-ray film revealed bilateral air trapping and right upper lobe consolidation. Sputum cultures were positive for adenovirus and Neisseria branhamella. Tracheobronchoscopic examination revealed tapering stenosis of the trachea beginning $2 \mathrm{~cm}$ beyond the vocal cords. Because it was impossible to advance the smallest telescope, the distal extent of the stenosis remained undefined. A thoracic computed tomographic scan demonstrated long-segment tracheal stenosis, but the carina and the main bronchi seemed normal. A tracheobronchographic examination was therefore not 


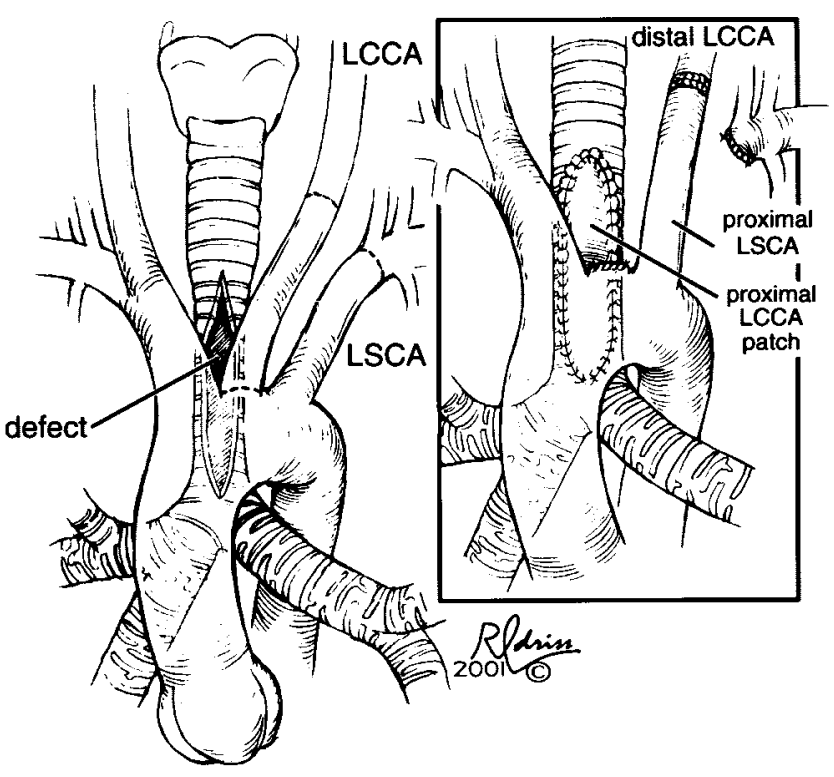

Figure 1. Anterior incision into the stenotic portion of the trachea. The anticipated incisions in the left common carotid artery (LCCA) and left subclavian artery (LSCA) are demonstrated by the dotted lines. Inset shows the completed tracheal repair with the patch of carotid artery and anastomosis of the left subclavian artery to reestablish perfusion of the distal left common carotid artery.

performed. Sudden uncontrollable respiratory deterioration with increased mean airway pressures, as well as ongoing sepsis, made the patient's condition highly unstable. Extracorporeal membrane oxygenation (ECMO) was unavailable at our institution, and emergency intratracheal balloon dilation or stenting seemed unreasonable without full anatomic knowledge of the lesion, making emergency surgical intervention inevitable.

We proceeded with salvage surgery in a 5.4-kg infant through an expeditious median sternotomy, with cannulation of the aorta and right atrial appendage, normothermic cardiopulmonary bypass, and a beating heart. Intraoperatively, it was possible to pass the bronchoscope beyond the proximal portion of the stenosis, revealing complete congenital tracheal rings extending down to the carina. This new evidence contradicted previous diagnostic information. Resection and end-to-end anastomosis or slide tracheoplasty seemed inappropriate. We opted for patch reconstruction, but the remaining pericardium after the hasty sternotomy appeared damaged, thin, and unsuitable. The infant's septic condition made xenopericardium or synthetic patch material undesirable, and no tracheal homograft was available. The trachea was opened anteriorly and longitudinally. As a last resort, an arterial conduit was selected for the graft material. The left common carotid artery was harvested from its aortic arch origin to its point of disappearance under the mandible (Figure 1). The artery was opened longitudinally and sutured to patch the tracheal defect with its endothelial side toward the airway lumen (Figure 1, inset). The left subclavian artery was fully mobilized to its thoracic exit, transected, and an end-to-end anastomosis to the stump of the left common carotid artery was performed (Figure 1, inset). To achieve stenting of the

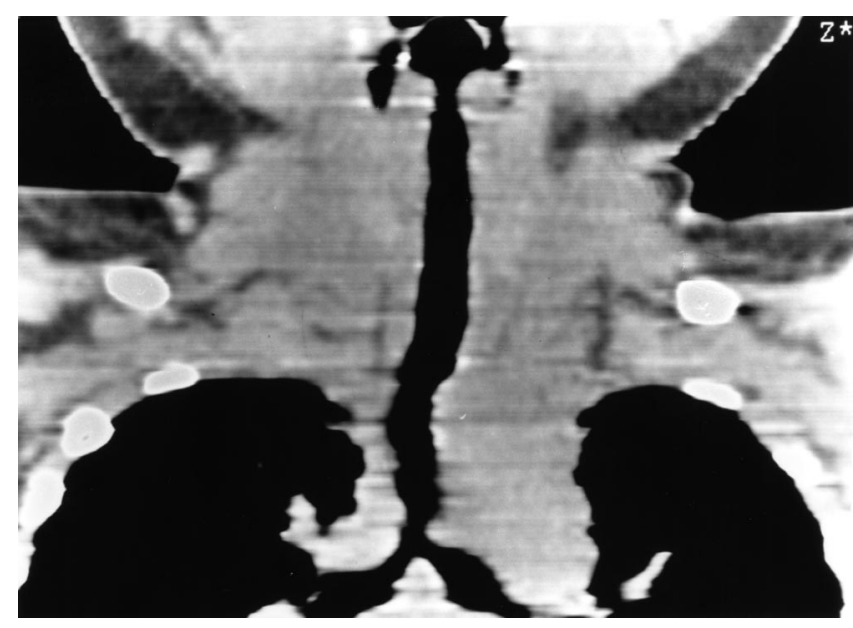

Figure 2. Three-dimensional computed tomographic scan of the reconstruction 2 weeks postoperatively. Note the patent but narrowed airway at the distal end of the repair. The flailing carotid artery patch subsequently became appropriately rigid with conservative management.

tracheal repair, the carotid patch was sutured to the aorta and adjacent mediastinal tissue, pulling it anteriorly toward the sternum. Intraoperative bronchoscopy confirmed a widely patent repair. Weaning from cardiopulmonary bypass, protamine, decannulation, and chest closure were without incident.

An uneventful postoperative course allowed extubation on postoperative day 7 and discharge from the hospital on postoperative day 16 after a satisfactory computed tomographic scan of the repair (Figure 2).

Current follow-up 3 years after the operation reveals a normally developing and neurologically intact child. She is completely free of symptoms and the physician parents find no justification in a follow-up bronchoscopic examination. The function, growth, and perfusion of her left arm are normal and comparable with the contralateral arm.

\section{Comment}

Diffuse stenosis from complete congenital tracheal rings remains a rare but challenging problem in neonates and infants, best diagnosed and managed in a multidisciplinary manner. ${ }^{1}$

Diagnostically, the indications for tracheobronchography should be appropriately widened. This was not performed in our patient, but would have allowed visualization of the distal extent of the stenosis, avoided unnecessary surprises, and permitted better planning for alternative patch materials than the one finally used.

Beyond surgical preference, ${ }^{2-4}$ tracheal anatomy and the availability of reconstruction materials may dictate the type and extent of repair. Although we were aware of the techniques of resection and end-to-end anastomosis, slide tracheoplasty, pericardial patch plasty, tracheal homograft, and autograft reconstruction, they were either unsuitable or impossible at the time of surgery. Excessive anastomotic tension would have made resection and end-to-end anastomosis, slide tracheoplasty, or autograft reconstruction hazardous. The remaining autologous pericardium was either damaged or excessively thin, and tracheal homograft was unavailable. 
The carotid artery was readily available, had the desired curvature, and was technically easy to handle. It is endothelialized, has sufficient tensile strength, and is autologous material. We were concerned enough to prefer sacrifice of the left subclavian artery for carotid artery reconstruction. More recent data from experience with ECMO in neonates and infants suggest the safety in temporarily using carotid arteries, because they are routinely repaired after weaning from ECMO. 5

We acknowledge the radical nature of this repair technique, but we present it as a last-resort option in emergency settings, when more conventional types of surgery are not possible because of misdiagnosis, unavailability, or inappropriateness of more standard reconstruction materials.

\section{References}

1. Dodge-Khatami A, Tsang VT, Roebuck DJ, Elliott MJ. Management of congenital tracheal stenosis: a multidisciplinary approach. Images Paediatr Cardiol. 2000;2:30-40.

2. Bryant LR, Eiseman B. Replacement of tracheobronchial defects with autogenous pericardium. J Thorac Cardiovasc Surg. 1964;48:733-40.

3. Tsang VT, Murday A, Gilbe C, Goldstraw P. Slide tracheoplasty for long-segment tracheal stenosis. Ann Thorac Surg. 1989;48:632-5.

4. Backer CL, Mavroudis C, Dunham ME, Holinger L. Repair of congenital tracheal stenosis with a free tracheal autograft. $J$ Thorac Cardiovasc Surg. 1998;115:869-74.

5. Goldman AP, Macrae DJ, Tasker RC, Edberg KE, Mellgren G, Herberhold $\mathrm{C}$, et al. Extracorporeal membrane oxygenation as a bridge to definitive tracheal surgery in children. J Pediatr. 1996;128:386-8.

\section{Notice from the American Board of Thoracic Surgery regarding trainees and candidates for certification who are called to military service related to the war on terrorism}

The Board appreciates the concern of those who have received emergency call to military service. They may be assured that the Board will exercise the same sympathetic consideration as was given to candidates, in recognition of their special contributions to their country, during the Viet Nam conflict and the Persian Gulf conflict with regard to applications, examinations, and interruption of training. If you have any questions about how this might affect you, please call the Board office at 847-475-1520.

Peter C. Pairolero, MD

Chair

The American Board of Thoracic Surgery 